\title{
Approximate Cross Channel Color Mapping from Sparse Color Correspondences
}

\author{
Hasan Sheikh Faridul ${ }^{1,2}$, Jurgen Stauder ${ }^{1}$, Jonathan Kervec ${ }^{1}$, Alain Trémeau ${ }^{2}$ \\ ${ }^{1}$ Technicolor Research \& Innovation, France \\ ${ }^{2}$ University of Saint Etienne, CNRS, UMR 5516, France \\ \{hasan.sheikh-faridul, jurgen.stauder\}etechnicolor.com \\ Alain.Tremeau@univ-st-etienne.fr
}

\begin{abstract}
We propose a color mapping method that compensates color differences between images having a common semantic content such as multiple views of a scene taken from different viewpoints. A so-called color mapping model is usually estimated from color correspondences selected from those images. In this work, we introduce a color mapping that model color change in two steps: first, nonlinear, channel-wise mapping; second, linear, cross-channel mapping. Additionally, unlike many state of the art methods, we estimate the model from sparse matches and do not require dense geometric correspondences. We show that well known cross-channel color change can be estimated from sparse color correspondence. Quantitative and visual benchmark tests show good performance compared to recent methods in literature.
\end{abstract}

\section{Introduction}

Many applications such as stereo imaging, multiple-view stereo, image stitching, photo-realistic texture mapping or color correction in feature film production, face the problem of color differences between images showing semantically common content. Possible reasons include: uncalibrated cameras, different camera settings, change of lighting condition, and differences between imaging workflows. Color mapping is a method that models such color differences between views to allow their compensation.

Color mapping is usually based on: matching corresponding features, computing color correspondences from those matched features and finally estimating a color map from the color correspondences. For instance, Figure 1 shows an example of color mapping. In the literature, finding color correspondences (first step of color mapping) is either based on sparse $[2,4,6,13,20,21,29,31]$ or on dense $[2,9]$ feature matching. To model the underlying color change (second step of color mapping), mostly channelwise $[2,6,9,13,27-31]$ color mapping models are used. Channel-wise means that color mapping models are independently estimated for each color channels (such as red, green and blue). In a channel-wise model, to estimate a channel of one view, contribution is taken only from the corresponding channel of the other view, that is:

$$
\begin{gathered}
R_{\text {view } 2}=f_{R}\left(R_{\text {view } 1}\right) \\
G_{\text {view } 2}=f_{G}\left(G_{\text {view } 1}\right) \\
B_{\text {view } 2}=f_{B}\left(B_{\text {view } 1}\right) .
\end{gathered}
$$

Channel-wise methods can be further categorized into linear $[2,28,30,31]$ or nonlinear $[6,9,12,14,21]$ models.

Channel-wise color mapping ignores the cross-channel influences, but is simpler and faster to estimate. However, in practice many color changes such as strong saturation or hue differences, due to illumination change or imaging devices change, do not follow channel-wise model assumption. In these situations, color change happens not only in red, green, and blue channels independently, but also in a cross-color-channel manner. Furthermore, these color changes are often nonlinear. To our knowledge, very few methods $[9,12,14]$ addressed cross-channel and nonlinear color change modeling - often not in the context of color mapping. In this work, we would like to answer the question whether it is possible and useful to estimate a crosschannel and nonlinear color map using only sparse color correspondences.

Like many methods from the literature, we start from the geometric feature correspondences. Then, our approach consists of two steps. In the first step, we robustly fit a nonlinear, channel-wise model. In the second step, we estimate a linear but cross-channel color model. The advantage of the first, channel wise step is that it captures the nonlinear color change. The advantage of the second step of linear but cross-channel model is that it is robust.

In order to compare our method with existing color map- 


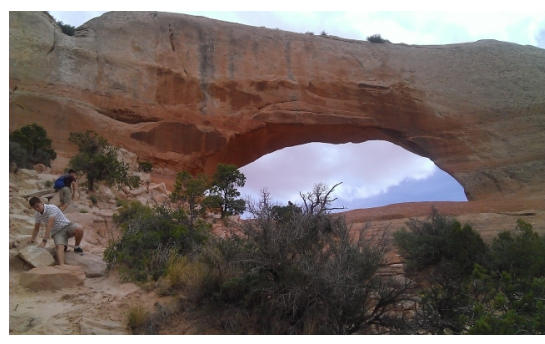

(A) Reference

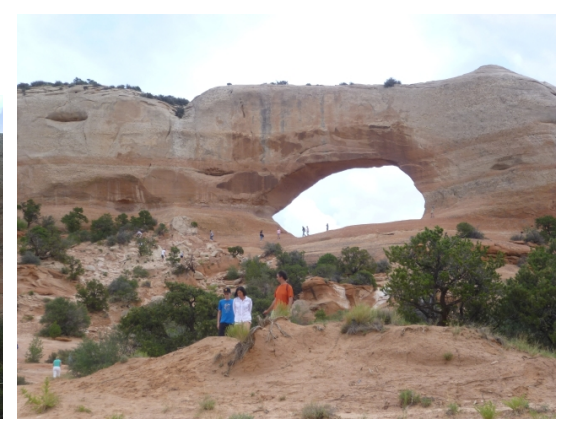

(B) Test

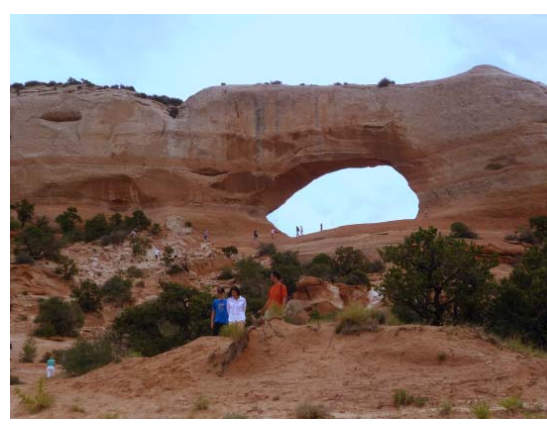

(C) Mapped by our method

Figure 1: An example of color mapping. (A) shows the reference view - colors that we want. (B) shows the test view colors that we want to change so that it matches with the reference. (C) shows result after our color mapping which matches closely with the reference.

ping methods, we use a qualitative (Section 4.1) as well as quantitative evaluation (Section 4.2). Since our model is neither constrained to channel-wise color changes nor requires dense color correspondences, a wider range of applications are possible.

\section{Related works}

A significant number of color mapping methods are available in the literature that may compensate color differences. These methods can be broadly classified into three main categories: first, geometric correspondence based methods $[2,4-6,9,10,13,20,22,29,31]$; second statistical distribution transfer based methods [7, 23-28]; third, user assisted methods [3, 15, 21].

Very few methods have addressed the cross-channel color change especially in the context of color mapping. For instance, computational color constancy algorithms [8] employ a cross-channel linear model, but do not apply necessarily to color mapping. Because, the objective of computational color constancy algorithms is to correct for illumination spectrum such that it looks like to be captured under canonical illuminant (such as CIE D65). Besides, scene illumination is required to be estimated or known as prior. Hacohen et al. [9] use also similar linear model after a channel-wise mapping.

Ilie and Adrian [12] address cross-channel change but from camera color calibration point of view. The authors estimate the cross-channel model from the 24 sample colors of GretagMacbeth ColorChecker. Unfortunately, that cannot be extended to color mapping since it requires a ColorChecker in the scene.

Although not necessarily color mapping, Kim et al. [14] propose an imaging model that can correct colors when camera settings are not "correct". A Radial Basis Function (RBF) based cross-channel color modeling is used in their work.
Despite significant contributions in related works, in the context of color mapping, no work provides a method that can model both cross channel and nonlinear color change between different viewpoints only from sparse color correspondences.

\section{A new cross-channel color mapping}

Our color mapping method is based on three steps. In the first step, we start by computing feature correspondences by SIFT [16]. We collect colors near to these feature correspondences that provide so-called color correspondences. Here, color correspondences define which colors from one view correspond to which colors of the other view. Note that, to generate the feature correspondences, other methods such as SURF [1], MSER [18] etc. can be utilized as well. In the second step, we robustly fit a channel-wise, constrained, nonlinear color mapping model. In the third step, we estimate a linear, but cross-channel model.

Let $C_{i} \leftrightarrow C_{i}^{\prime}$ be the color correspondences with $i$ its index, that is, $C_{i}$ is a color from one view that corresponds to the color $C_{i}^{\prime}$ from the other view. Let us assume three color channels, that is, $C \epsilon\{R, G, B\}$, where $R, G, B$ stands for red, green, and blue color correspondences, respectively.

Let us also assume, $M$ be the number of color correspondences. Therefore, both $C$ and $C^{\prime}$ are $M \times 3$ matrices. Each $C_{i} \leftrightarrow C_{i}^{\prime}$ can be read as a 3-tuple correspondence, that is, $(R, G, B) \leftrightarrow\left(R^{\prime}, G^{\prime}, B^{\prime}\right)$.

From these color correspondences, the color mapping model $f_{C}$ is estimated such that it minimizes the following color differences:

$$
\min \sum_{i=1}^{M}\left(C_{i}-f_{C}\left(C_{i}^{\prime}\right)\right)^{2} .
$$

In this work, we estimate $f_{C}$ in two steps: first, channelwise nonlinear estimation (Section 3.1); second, cross- 
channel linear estimation (Section 3.2). A pseudo-code of the whole proposed method is presented in Algorithm 1

\subsection{Step1: Channel-wise estimation}

In this section, using the color correspondences, we estimate the color mapping model, $f_{C}$ which describes and compensates the color differences in a channel-wise manner.

Before estimation, for regularization purpose, we add two imaginary white and black color correspondences. Since we assume high quality content and no clipping, for a $P$-bit image, we add $\left(\begin{array}{llll}1 & 1 & 1\end{array}\right) \leftrightarrow\left(\begin{array}{lll}1 & 1 & 1\end{array}\right)$ and $\left(2^{P} 2^{P} 2^{P}\right) \leftrightarrow$ $\left(\begin{array}{lll}2^{P} & 2^{P} & 2^{P}\end{array}\right)$ in $C_{i} \leftrightarrow C_{i}^{\prime}$. We do this to account for often missing very dark and very bright colors in high quality pictures. This also help the estimation to avoid extrapolation. We estimate the color correction model in the following three main steps.

In the first step, for each color channel, we estimate a piece-wise cubic spline from the list of color correspondences, $C_{i} \leftrightarrow C_{i}^{\prime}$ having $K$ knots. We set constraint during the estimation so that the estimated curves are monotonic, which is common in recent works [9, 11]. Similar to [9], we use $K=6$ so that the model can capture some nonlinear changes. We expect this model to be approximate (since channel-wise) but robust.

In the second step, for each color channel, we compute the standard deviation of residuals $\sigma_{C}$. If the residual of a color correspondence is larger than $5 * \sigma_{C}$ for any color channel, this color correspondence is considered as outlier. Then, it is removed from the list of color correspondences.

Lastly, in the third step, we again estimate the model for each color channel, but from the "outlier-free" color correspondences.

\subsection{Step2: Cross channel estimation}

In this step, we estimate cross-channel color mapping model. Cross-channel change such as saturation change due to illuminant spectrum change can not be compensated by channel-wise model only. Therefore, we describe the remaining cross channel color changes by a linear model. Cross channel color change can be nonlinear. So, we propose here an approximate but robust model.

Let $\hat{C}$ denote colors after applying the channel wise mapping on $C$. The resulting color correspondences is, $\hat{C}_{i} \leftrightarrow C_{i}^{\prime}$. Now, we estimate the cross-channel model parameters, $\theta$ by solving the following normal equation, where $\theta$ is a $3 \times 3$ matrix.

$$
\theta=\left(\hat{C}^{T} * \hat{C}\right)^{-1} * \hat{C}^{T} *\left(C^{\prime}\right)
$$

\section{Experiment}

In this section, we present experimental results obtained with our method. We also compare it with the color correc-

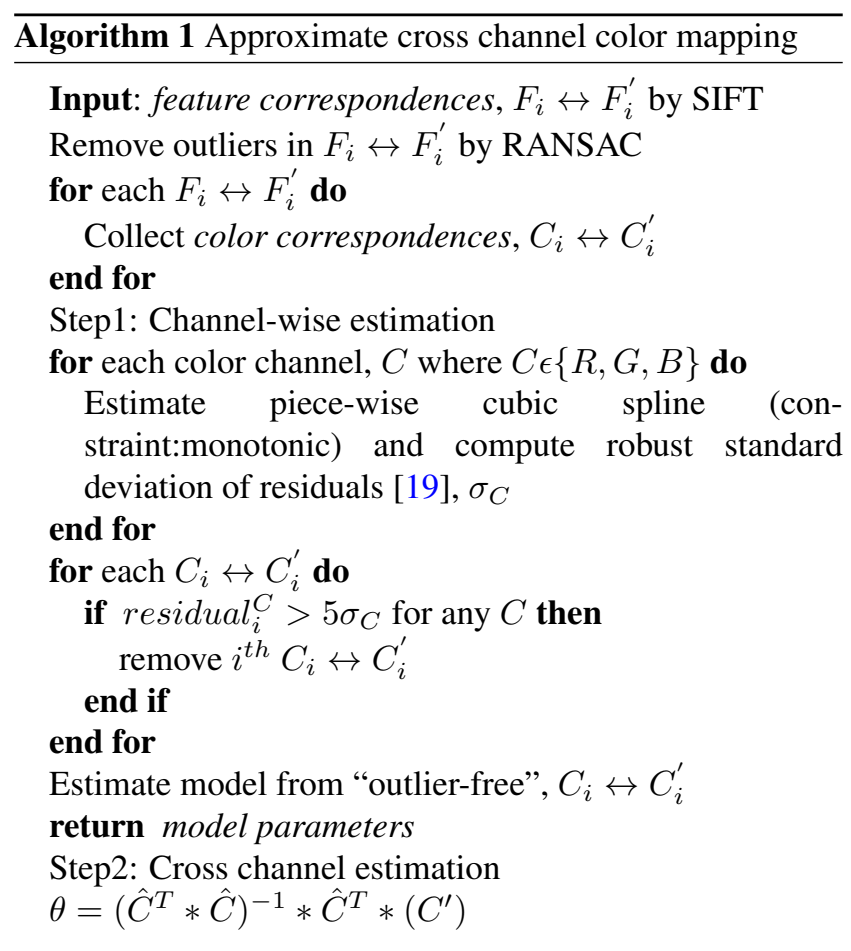

tion methods from the state of the art: Hacohen et al. [9], Pitie et al. [24]; and, Reinhard et al. [27]; For these methods, we have used the original codes/executables provided by the authors.

In all following experiments, given an image as example (i.e. reference), color mapping is applied on the test images. We first show qualitative (Section 4.1) and then quantitative (Section 4.2) results. We particularly focus on test images having nonlinear and cross-channel color change.

\subsection{Qualitative results}

\subsubsection{Differences in imaging devices}

Differences in imaging devices can lead to complex color change. Possible reasons include nonlinear processing [14] applied by the camera or differences in optics, sensors, different color filters and different color primaries. For this experiment, we captured images using different consumer imaging devices that are mentioned in the Table 1. In Figure 2, (A), (C), and (E) show three reference images, whereas (B), (D), and (F) show the corresponding test images. Given a reference image, we apply color mapping on the test image. Resulting images after color mapping are shown in Figure 3. Red rectangles point out the remaining errors such as the artifacts or strong remaining luminance differences. From visual inspection, we observe that in Figure 3, method from Pitie et al. [24] and Reinhard et al. [27] performs comparatively worse than Hacohen et al. [9] and our method. 
Table 1: Different imaging devices used in experiment (Section 4.1.1)

\begin{tabular}{lr}
\hline Device\# & Device name \\
\hline Device1 & Panasonic DMC FS-11 \\
Device2 & HTC phone (Model QCAM-AA) \\
\hline
\end{tabular}

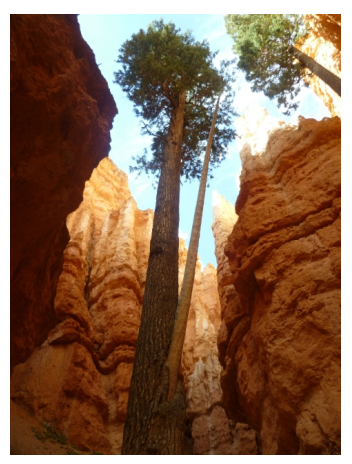

(A) Reference: Device\#1

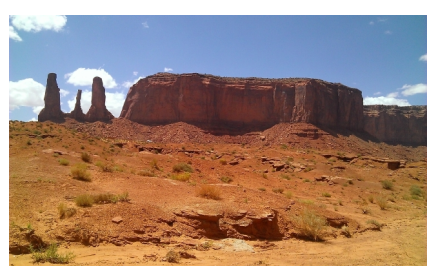

(C) Reference: Device\#2

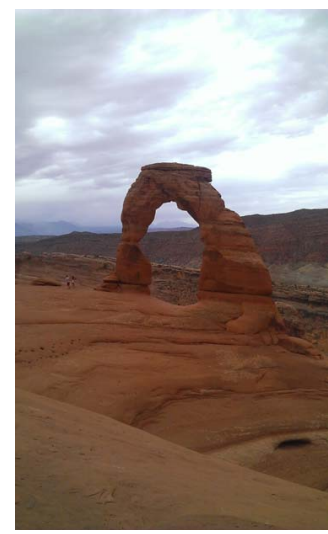

(E) Reference: Device\#2

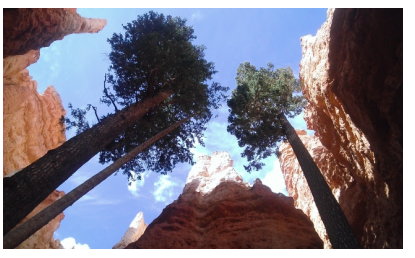

(B) Test: Device\#2

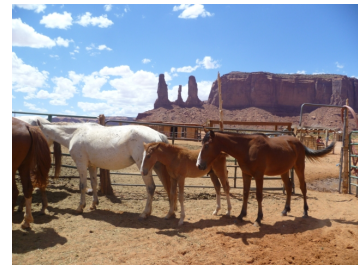

(D) Test: Device\#1

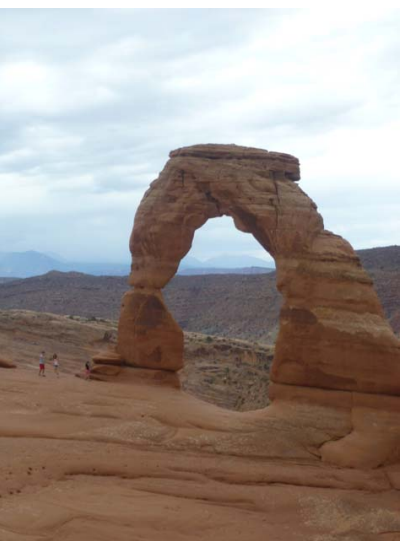

(F) Test: Device\#1
Figure 2: Input images for color mapping captured by different imaging devices. List of imaging devices are mentioned in Table 1. See color mapping results for these images in Figure 3

\subsubsection{Differences in illumination spectrum}

In this section, we qualitatively compare color mapping performance in case of illuminant spectrum change. Original images are shown in Figure 4. In this figure, given the im- age under reference illuminant, we apply color mapping on the test image that we acquired under "Blue", "Red', and "Cyan" illuminants. We used led panels from Elation (TVL2000), and color spots from Spectral (M800 Tour) \& Flat Par (TRi7x). We controlled these light sources by a MAYA plugin. These images were captured by a CANON 7D camera. The results after color mapping are presented in Figure 5. Like previous experiment, the method from Pitie et al. [24] results into clearly visible artifacts in Figure 5. The method from Reinhard et al. [27] also fails because their simple model of scaling mean and variance cannot capture illuminant spectrum change. The method from Hacohen et al. [9] also fails. For example, in the first column of Figure 5, notice the remaining color differences on the skin tone of the person. On the other hand, the fourth column of Figure 5 shows that our method visually performs well compared to other methods.

\subsection{Quantitative results}

Since qualitative results might be subjective and difficult to evaluate, in this section, we provide quantitative results of our color mapping method. Since the method from Pitie et al. [24] creates disturbing artifacts, we no longer report performance for this method in this section. We continue to compare our method with the method from Hacohen et al. [9] and Reinhard et al. [27]. To report quantitatively, ground truth is required.

Ground truth: Let us consider a static scene acquired under two different illumination conditions (illum 1 and illum2) and from two different viewpoints (viewpoint1 and viewpoint2). Under viewpoint1 and illuminant illum1, a first image (Img1) is captured. Next, under viewpoint 2 and illuminant illum 1 , a second image (Img2) is captured. As $I m g 1$ and $I m g 2$ are taken under the same illumination condition (illum 1 ) and that they represent the same scene and we assume lambertian surface, their colors are consistent even though the two viewpoints are different. A third image ( $\operatorname{Img} 3$ ) is acquired under the same viewpoint as for the second image (i.e. viewpoint2) but under illuminant illum2. As $I m g 1$ and $I m g 3$ are taken under different illumination conditions (illum 1 vs. illum2), their colors are different. Now, if we consider that $I m g 1$ is the reference view and $I m g 3$ is the test view, then $I m g 2$ is the ground truth.

Evaluation metric: We evaluate the performance using the remaining color differences with respect to the ground truth. Taking the average for the whole image provides the overall remaining difference,

$$
\mu_{\Delta R G B}=\frac{\sum_{i, j=1}^{M, N} \Delta R G B}{M * N}
$$




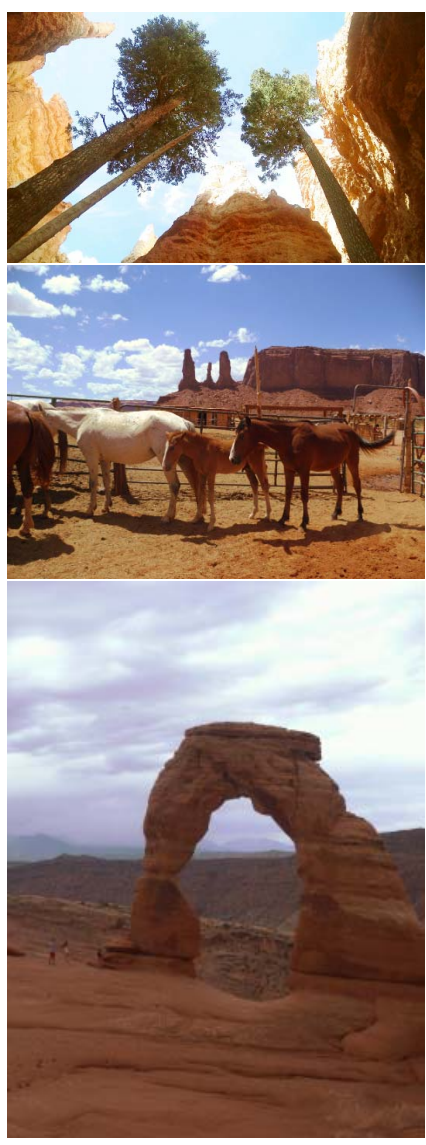

(A) Hacohen et al. [9]

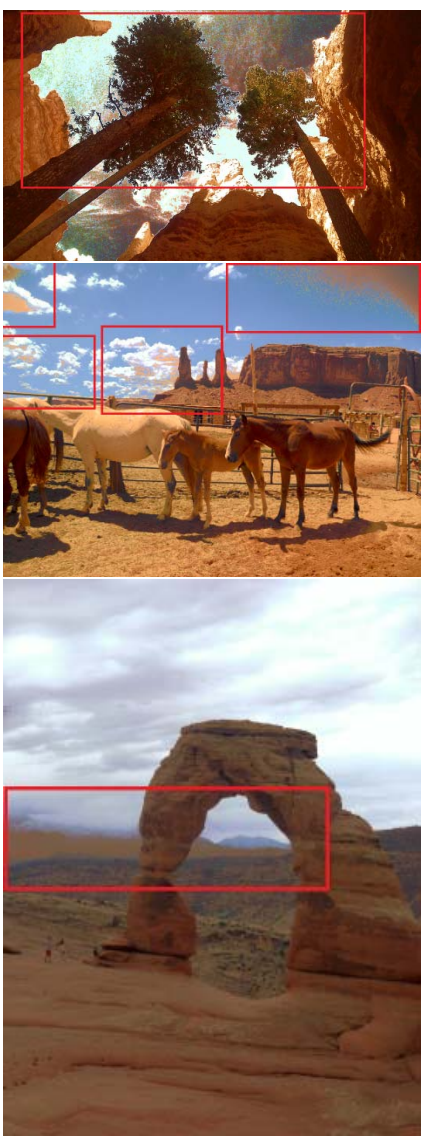

(B) Pitie et al. [24]
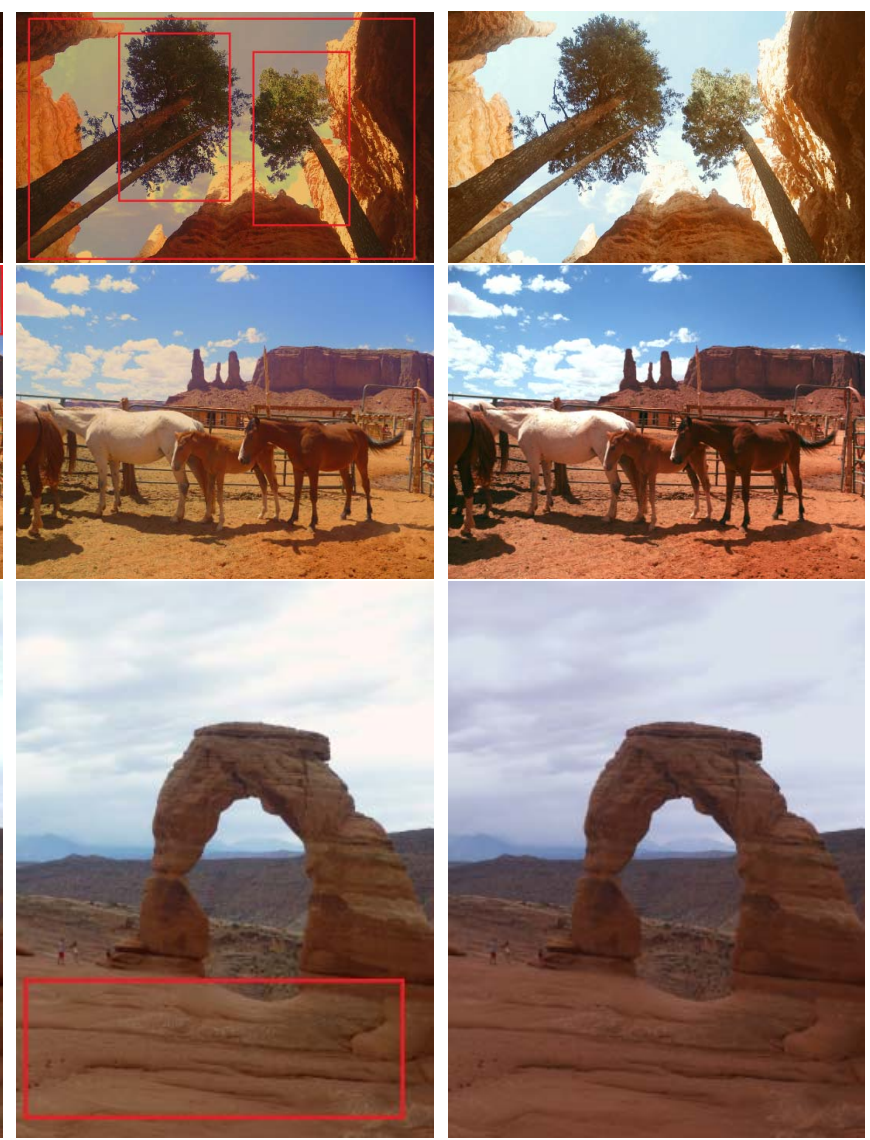

(C) Reinhard et al. [27]

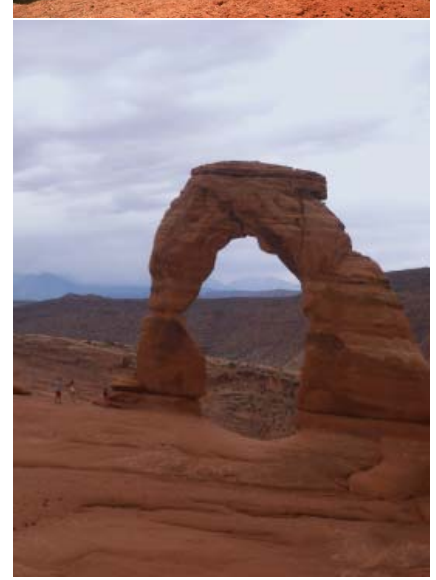

(D) our result

Figure 3: Resulting images after color mapping. See input images in Figure 2. Red rectangles show artifacts or strong remaining luminance differences. Method from Pitie et al. [24] results into clearly visible artifacts. Method from Reinhard et al. [27] over-compensate or under compensates. On the other hand, our method as well as method from Hacohen et al. [9] comparatively well compensate the color differences for these test images.

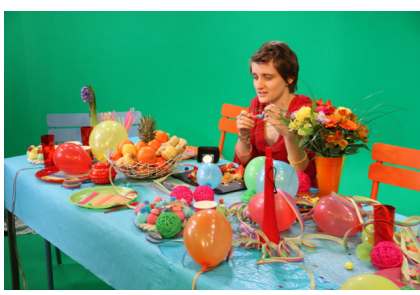

(A) Reference illuminant

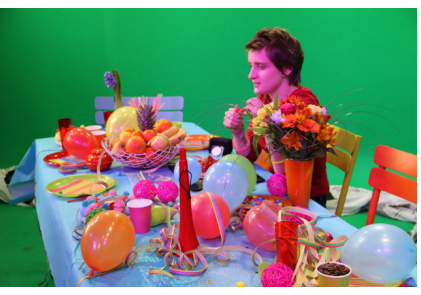

(B) "Blue" illuminant

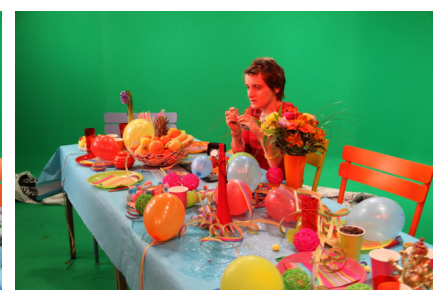

(C) "Red" illuminant

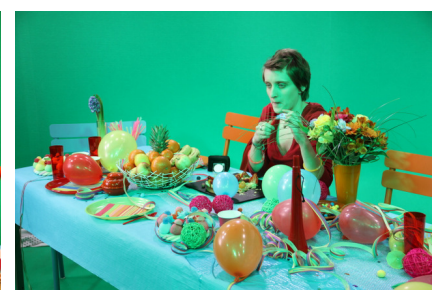

(D) "Cyan" illuminant

Figure 4: Input images for color mapping acquired under several illuminant spectra and from different viewpoints. These input images have strong cross-channel color differences. See the results after color mapping in Figure 5.

Here, $M * N$ is the total number of pixels in the image. $\triangle R G B(i, j)$ is the euclidean distance in RGB color space between mapped color from test view and ground truth view at $(i, j)$ pixel location. We compare several color mapping methods by comparing their $\mu_{\Delta R G B}$. Note that, other metrics such as perceptual metric CIE $\Delta E 00$ [17] can be used as well.

Let us first report the case of illumination spectrum and intensity change. Figure 6 provides test images having ground truth. In these test images, there is a change in spectrum, intensity as well as viewpoint. Like previous experiments, given the reference images, color mapping is applied 


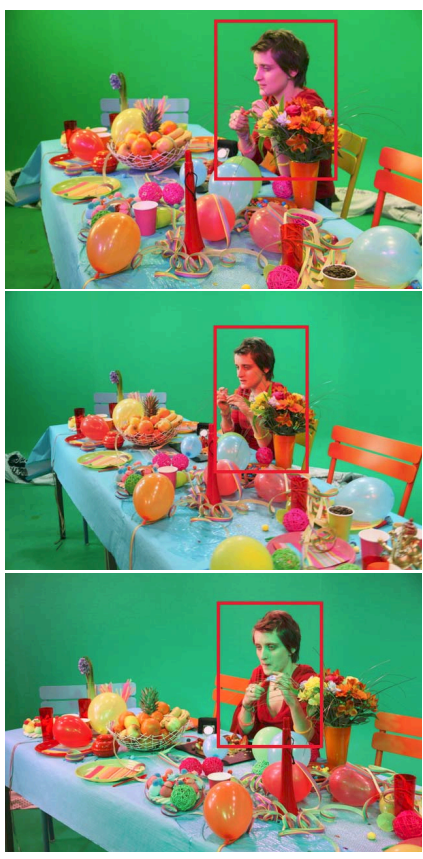

(A) Hacohen et al. [9]
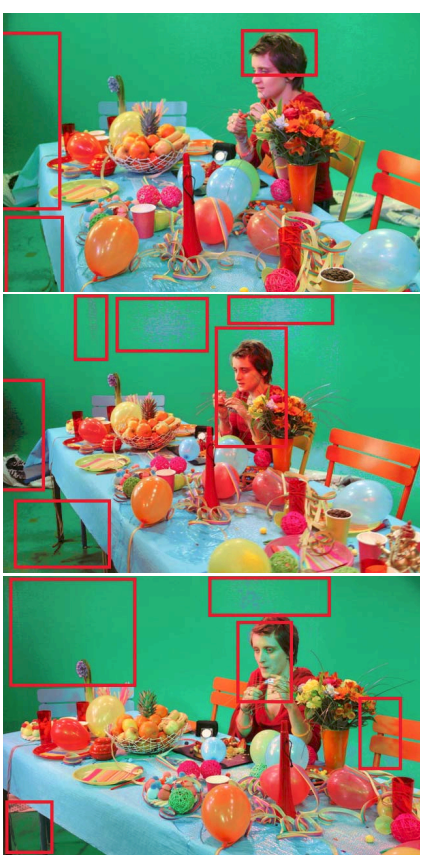

(B) Pitie et al. [24]
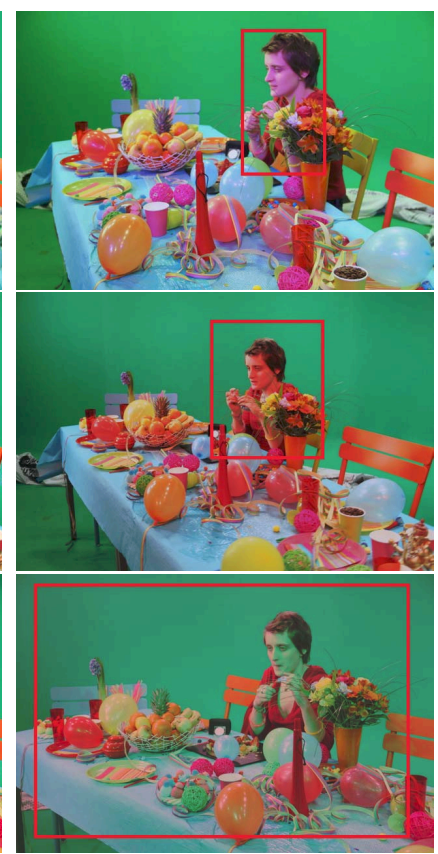

(C) Reinhard et al. [27]

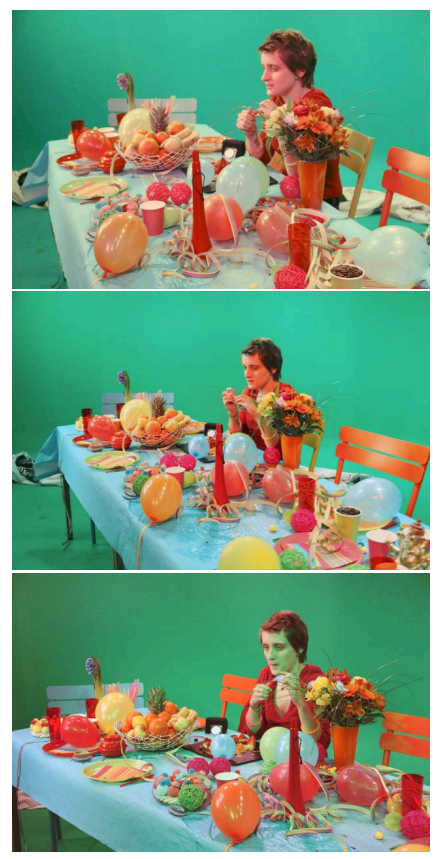

(D) Our result

Figure 5: Results after color mapping. Input (reference and test) images having illumination spectrum difference are shown in Figure 4. Visually compare these results with Figure 4 (A). Like previous case, method from Pitie et al. [24] have shown strong artifacts. Method from Reinhard et al. [27] has also failed since their model is too simple to capture such illuminant spectrum change. Method from Hacohen et al. [9] has also failed as the resulting difference is visible on the skin tone. We show red rectangles to point out artifacts or strong remaining luminance differences.

to the test images. Error maps representing by remaining $\triangle R G B$ are shown in Figure 7. These error maps quantitatively show and localize the remaining error after color mapping where the gray values correspond to $\triangle R G B$. The lower the $\triangle R G B$, the darker is the error maps, the better is the performance. Quantitative result in Figure 7 confirms that our method compensate colors better than both Hacohen et al. [9] and Reinhard et al. [27]. This proves the robustness of our method to strong cross-channel and nonlinear color change.

We now look at quantitative results in case of more complex color change. Figure 8 shows the reference image under "white" illuminant, but the test images under "yellowish" illuminant. Furthermore, in these test images of Figure 8 (A) to (E), we changed the camera white balance from $7000 \mathrm{k}$ to $3000 \mathrm{~K}$ during the capture with a CANON 7D camera. This produces yellowish (Figure 8 (A)) to blue-wish (Figure 8 (E)) images.

Given the reference image as example, color mapping is applied on the test images. After that, we compare the color mapping results with the ground truth that produce error maps like the previous experiment. For each method, and each test image of Figure 8, we calculate $\mu_{\triangle R G B}$ using Eq. 4. This produces the error bar which are shown in Figure 9. Lines on the error bars correspond to 1 standard deviation. The error bars in Figure 9 shows significant improvement of our method compared to other mentioned methods from $7000 \mathrm{~K}$ to $4000 \mathrm{~K}$. This proves the robustness of our method to strong cross-channel as well as nonlinear color change.

\subsection{Discussion}

Our first observation from these experiments is that method from Pitie et al. [24] as well as Reinhard et al. [27] can compensate simple color changes. However, in case of a complex color change such as cross-channel change (e.g. illumination spectrum), these methods fail. Particularly Pitie et al. [24] often results into disturbing visual artifacts. Reinhard et al. [27] fails because of their too simple model that can not capture nonlinear and cross channel color change. As a result, method from Reinhard et al. [27] often results over-compensation or under-compensation especially in case of nonlinear and cross channel color change. The advantage of both Pitie et al. [24] and Reinhard et al. [27] is that their method do not require geometric feature correspondences.

Method from Hacohen et al. [9] perform comparatively better both in terms of color compensation and robustness 


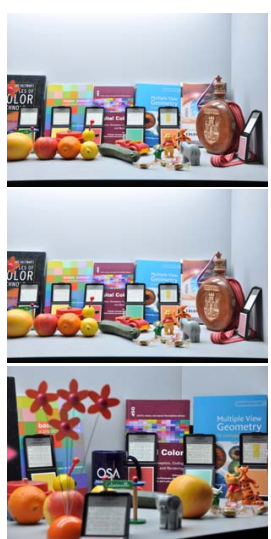

(A) Reference

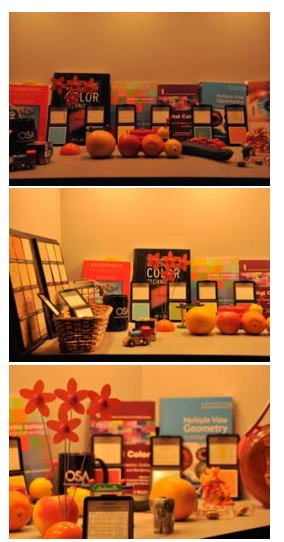

(B) Test

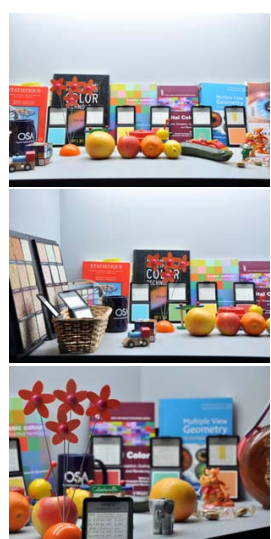

(C) Ground truth
Figure 6: Input images for color mapping having ground truth. First column shows the reference, second column shows the test and the third column shows the corresponding ground truth. Between (A) and (B), there is a change in spectrum and intensity. Between (D) and (E), there is a change in strong viewpoint and spectrum. Lastly, between (G) and (H), there is a change in focus in foreground vs. focus in background with a change in spectrum.

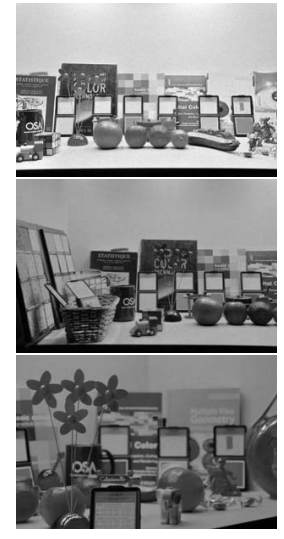

Hacohen et al. [9]

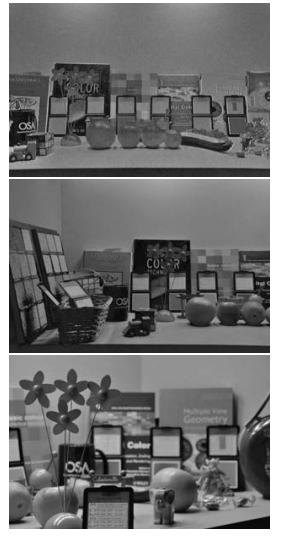

Reinhard et al. [27]

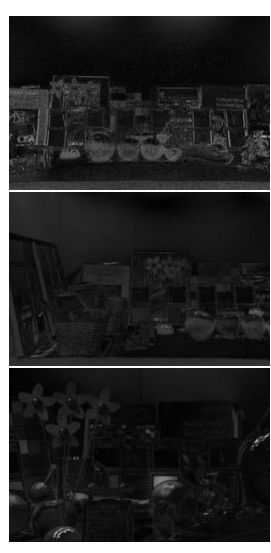

Our result
Figure 7: Error maps produced by $\triangle R G B$ that localize the remaining errors after comparing with the ground truth. The lower the $\triangle R G B$, the darker is the error maps, the better is the performance.

compared to both Pitie et al. [24] and Reinhard et al. [27]. However, the method from Hacohen et al. [9] also fails in case of cross channel change such as illuminant spectrum change. This is because, their method failed to make feature correspondences.

On the other hand, our method succeeds, thanks to its robustness to strong nonlinear and cross channel color change. This robustness comes from the robust estimation of the nonlinear channel-wise model followed by the linear cross- channel model.

\subsection{Limitations}

Since the proposed method depends on feature matching, color mapping scope is limited to scenes where such matching is possible. We also noticed that feature matching (sparse of even dense) might fail to make correspondences for colors in the homogeneous part of the scene. Besides, in our method, we estimate a single map for the whole image. Therefore, our method might be limited to very strong local color differences such as local hue shift caused by drastic changes of illumination direction as well as spectrum change or those produced by manual image editing.

\subsection{Conclusions}

In this paper, we have presented a color mapping method for the compensation of color differences between images utilizing sparse color correspondences.

Our color mapping method first selects color correspondences using feature matches. Then we eliminate outliers and fit a nonlinear channel-wise model. To model the crosschannel change, we estimate a simple linear model.

We conducted experiments with images having illuminant spectrum difference, imaging device difference and imaging settings difference (e.g. white balance). Experimental results show the robustness of our method compared to the state of the art. This initial result is very promising since our method utilize only sparse color correspondences. We conclude that dense color correspondences (like Hacohen et al. [9]) are not necessary for color mapping. In this work, we proved that robust color mapping can be estimated from sparse color correspondences. The gain in performance of our method comes from robust estimation of nonlinear channel-wise model and the robust, linear crosschannel model.

\section{References}

[1] H. Bay, A. Ess, T. Tuytelaars, and L. Van Gool. Speeded-up robust features (SURF). CVIU, 110(3):346-359, 2008. 2

[2] Y. Chen, K. Ma, and C. Cai. Histogram-offset-based color correction for multi-view video coding. In Proc. of ICIP, pages 977-980, 2010. 1,2

[3] O. Dalmau-Cedeno, M. Rivera, and P. P. Mayorga. Computing the $\alpha$-channel with probabilistic segmentation for image colorization. In Proc. of ICCV, pages 1-7. IEEE, 2007. 2

[4] C. Doutre and P. Nasiopoulos. Color correction preprocessing for multiview video coding. IEEE Trans. on Circuits and Systems for Video Technology, 19(9):1400-1406, 2009. 1, 2

[5] H. S. Faridul, J. Stauder, and A. Trémeau. Robust color correction for stereo. In Proc. of Conference for Visual Media Production (CVMP), pages 101-108, 2011.

[6] H. S. Faridul, J. Stauder, and A. Trémeau. Optimization of sparse color correspondences for color mapping. In Proc. of Color and Imaging Conference (CIC), 2012. 1, 2

[7] D. Freedman and P. Kisilev. Object-to-object color transfer: Optimal flows and smsp transformations. In Proc. of CVPR, pages 287 -294, jun. 2010. 2 


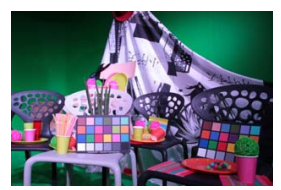

Reference:7000K

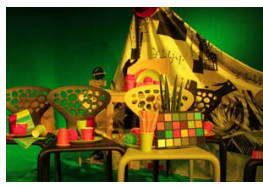

(A) Test:7000K

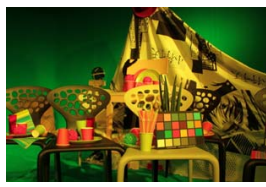

(B) Test:6000K

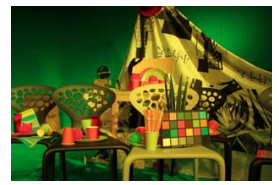

(C) Test:5000K

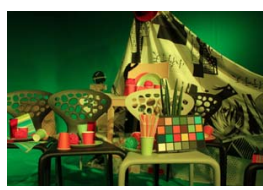

(D) Test:4000K

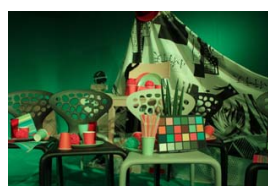

(E) Test:3000K

Figure 8: Test images having cross channel color change. Reference image is acquired under "white" light, whereas test images are acquired under "yellow" light. Then, for the rest of the test images, white balance in the camera (CANON 7D) is changed from $7000 \mathrm{~K}$ to $3000 \mathrm{~K}$ - producing a yellowish to blue-wish change. We compare the result after color mapping for several methods and produce the error bars shown in Figure 9.

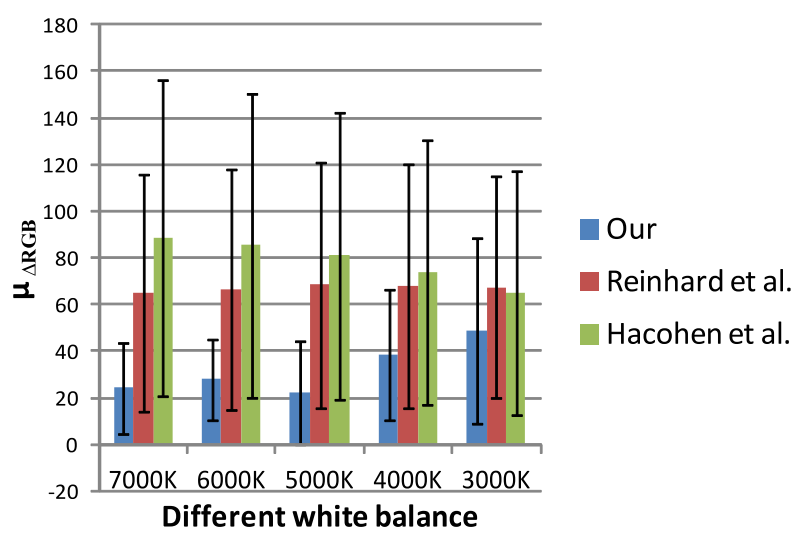

Figure 9: Quantitative results by error bars for the test images shown in Figure 8. Line on bars corresponds to 1 standard deviation. Method from both Hacohen et al. [9] and Reinhard et al. [27] perform poorly. Method from Hacohen et al. [9] fails since their method could not make correspondences in the presence of strong illumination change. Method from Reinhard et al. [27] fails due to their too simple model which cannot capture complex color changes. On the other hand, our method improves the results significantly due to its robustness to strong illumination and crosschannel color change.

[8] T. Gevers, A. Gijsenij, J. Van de Weijer, and J.-M. Geusebroek. Color in computer vision: Fundamentals and applications, volume 24 . Wiley. com, 2012. 2

[9] Y. HaCohen, E. Shechtman, D. B. Goldman, and D. Lischinski. Nonrigid dense correspondence with applications for image enhancement. Proc. of ACM SIGGRAPH, 30(4):70:1-70:9, 2011. 1, 2, 3, $4,5,6,7,8$

[10] Y. HaCohen, E. Shechtman, D. B. Goldman, and D. Lischinski. Optimizing color consistency in photo collections. ACM Transactions on Graphics (TOG), 32(4):38, 2013. 2

[11] J. Hu, O. Gallo, and K. Pulli. Exposure stacks of live scenes with hand-held cameras. In Proc. of ECCV, pages 499-512. Springer, 2012. 3

[12] A. Ilie and G. Welch. Ensuring color consistency across multiple cameras. In Proc. of ICCV, volume 2, pages 1268-1275, 2005. 1, 2

[13] S. Kim and M. Pollefeys. Robust radiometric calibration and vignetting correction. IEEE Trans. on PAMI, pages 562-576, 2007. 1,
[14] S. J. Kim, H. T. Lin, Z. Lu, S. Sabine, et al. A new in-camera imaging model for color computer vision and its application. IEEE Trans. on PAMI, pages 2289-2302, 2012. 1, 2, 3

[15] D. Lischinski, Z. Farbman, M. Uyttendaele, and R. Szeliski. Interactive local adjustment of tonal values. ACM Transactions on Graphics (TOG), 25(3):646-653, 2006. 2

[16] D. Lowe. Distinctive image features from scale-invariant keypoints. Int'l J. Computer Vision, 60(2):91-110, 2004. 2

[17] M. Luo, G. Cui, and B. Rigg. The development of the CIE 2000 colour-difference formula: CIEDE2000. Color Research \& Application, 26(5):340-350, 2001. 5

[18] J. Matas, O. Chum, M. Urban, and T. Pajdla. Robust wide-baseline stereo from maximally stable extremal regions. Image and Vision Computing, 22(10):761-767, 2004. 2

[19] H. Motulsky and R. Brown. Detecting outliers when fitting data with nonlinear regression-a new method based on robust nonlinear regression and the false discovery rate. BMC bioinformatics, 7(1):123, 2006. 3

[20] M. Oliveira, A. Sappa, and V. Santos. Unsupervised Local Color Correction for Coarsely Registered Images. In Proc. of CVPR, pages 201-208, June 21-23 2011. 1, 2

[21] T. Oskam, A. Hornung, R. W. Sumner, and M. Gross. Fast and stable color balancing for images and augmented reality. In Proc. of 3DIMPVT, pages 49-56, 2012. 1,2

[22] T. Owens, K. Saenko, A. Chakrabarti, Y. Xiong, T. Zickler, and T. Darrell. Learning object color models from multi-view constraints. In Proc. of CVPR, pages 169-176, 2011. 2

[23] N. Papadakis, E. Provenzi, and V. Caselles. A variational model for histogram transfer of color images. IEEE Trans. on Image Processing, 20(6):1682-1695, 2011. 2

[24] F. Pitié, A. Kokaram, and R. Dahyot. Automated colour grading using colour distribution transfer. CVIU, 107(1-2):123-137, 2007. $3,4,5,6,7$

[25] T. Pouli and E. Reinhard. Progressive color transfer for images of arbitrary dynamic range. Computers \& Graphics, 35(1):67-80, 2011.

[26] J. Rabin, J. Delon, and Y. Gousseau. Removing Artefacts From Color and Contrast Modifications. IEEE Transactions on Image Processing, 20:3073-3085, 2011.

[27] E. Reinhard, M. Adhikhmin, B. Gooch, and P. Shirley. Color transfer between images. IEEE Computer Graphics and Applications, 21(5):34-41, 2001. 1, 3, 4, 5, 6, 7, 8

[28] Y. Tai, J. Jia, and C. Tang. Local color transfer via probabilistic segmentation by expectation-maximization. In Proc. of $C V P R$, volume 1, pages 747-754, 2005. 1, 2

[29] M. P. Tehrani, A. Ishikawa, S. Sakazawa, and A. Koike. Iterative colour correction of multicamera systems using corresponding feature points. Journal of Visual Communication and Image Representation, 21(5-6):377 - 391, 2010. 1, 2

[30] G. Y. Tian, D. Gledhill, D. Taylor, and D. Clarke. Colour correction for panoramic imaging. In Proceedings. of Information Visualisation, pages $483-488,2002.1$

[31] Q. Wang, X. Sun, and Z. Wang. A robust algorithm for color correction between two stereo images. In Proc. of ACCV, volume 5995, pages 405-416, 2010. 1, 2 